\title{
Illuminating Radiogenomic Characteristics of Glioblastoma Multiforme through Integration of MR Imaging, Messenger RNA Expression, and DNA Copy Number Variation
}

\author{
Neema Jamshidi, MD,PhD, Maximilian Diehn, MD,PhD, Markus Bredel, MD,PhD, and \\ Michael D. Kuo, MD \\ Department of Radiological Sciences, UCLA School of Medicine, Box 951721, CHS 17-135, Los \\ Angeles, CA 90095-1721 (N.J., M.D.K.); Institute for Stem Cell Biology and Regenerative \\ Medicine, Stanford University, Palo Alto, Calif (M.D.); and Department of Radiation Oncology, \\ University of Alabama at Birmingham School of Medicine, Birmingham, Ala (M.B.).
}

\section{Abstract}

Purpose: To perform a multilevel radiogenomics study to elucidate the glioblastoma multiforme (GBM) magnetic resonance (MR) imaging radiogenomic signatures resulting from changes in messenger RNA (mRNA) expression and DNA copy number variation (CNV).

\begin{abstract}
Materials and Methods: Radiogenomic analysis was performed at MR imaging in 23 patients with GBM in this retrospective institutional review board-approve HIPAA-compliant study. Six MR imaging features - contrast enhancement, necrosis, contrast-to-necrosis ratio, infiltrative versus edematous T2 abnormality, mass effect, and subventricular zone (SVZ) involvement-were independently evaluated and correlated with matched genomic profiles (global mRNA expression and DNA copy number profiles) in a significant manner that also accounted for multiple hypothesis testing by using gene set enrichment analysis (GSEA), resampling statistics, and analysis of variance to gain further insight into the radiogenomic signatures in patients with GBM.
\end{abstract}

\begin{abstract}
Results: GSEA was used to identify various oncogenic pathways with MR imaging features. Correlations between 34 gene loci were identified that showed concordant variations in gene dose and mRNA expression, resulting in an MR imaging, mRNA, and CNV radiogenomic association map for GBM. A few of the identified gene-to-trait associations include association of the contrast-to-necrosis ratio with $K L K 3$ and $R U N X 3$; association of $\mathrm{SVZ}$ involvement with Ras oncogene family members, such as $R A P 2 A$, and the metabolic enzyme $T Y M S$; and association of vasogenic edema with the oncogene FOXP1 and PIK3IP1, which is a member of the PI3K signaling network.
\end{abstract}

\footnotetext{
Address correspondence to M.D.K. (michaelkuo@mednet.ucla.edu).

Author contributions:

Guarantors of integrity of entire study, N.J., M.D.K.; study concepts/study design or data acquisition or data analysis/interpretation, all authors; manuscript drafting or manuscript revision for important intellectual content, all authors; approval of final version of submitted manuscript, all authors; literature research, N.J., M.D.K.; clinical studies, M.D., M.D.K.; statistical analysis, N.J., M.D.K.; and manuscript editing, N.J., M.D.K.
}

Disclosures of Conflicts of Interest: N.J. No relevant conflicts of interest to disclose. M.D. No relevant conflicts of interest to disclose. M.B. No relevant conflicts of interest to disclose. M.D.K. No relevant conflicts of interest to disclose. 
Conclusion: Construction of an MR imaging, mRNA, and CNV radi-ogenomic association map has led to identification of MR traits that are associated with some known high-grade glioma biomarkers and association with genomic biomarkers that have been identified for other malignancies but not GBM. Thus, the traits and genes identified on this map highlight new candidate radiogenomic biomarkers for further evaluation in future studies.

Radiogenomics represents the evolution of the radiology-pathology correlation from the tissue level to the subcellular level. Radiogenomic analysis, which is the identification of imaging traits that correspond to different molecular phenotypes with clinical and biologic relevance, has been demonstrated in different tissues and with different imaging modalities (1-3). Different magnetic resonance (MR) imaging radiogenomic signatures have been identified successfully for glioblastoma multiforme (GBM); these have been reflective of different cellular processes and can be used to predict clinical outcomes by using gadolinium-enhanced MR imaging and genomescale messenger RNA (mRNA) microarray profiling (1). There continues to be an increasing number of studies in which researchers explore and define the genomic characteristics of MR imaging features in patients with GBM (4-7). The predictive capabilities of these signatures lead to exciting and compelling implications, both for understanding how molecular programs are revealed at tissue and imaging levels and for potentially understanding the molecular pathogenesis of a given tumor.

The causal factors underlying changes in gene expression patterns have not yet been fully elucidated in patients with GBM. Correlation-based studies do not necessarily imply causality in relationships; however, identifying correlations in independent data types and identifying the overlap between the correlations may present an approach that can be used to identify functionally related genes that are predictive of different imaging phenotypes. Specifically, similar genes with changes in both mRNA expression and DNA copy number variation $(\mathrm{CNV})$ that are correlated with particular core imaging features are strongly suggestive of a causal link between gene dose and tumor appearance.

The use of imaging features derived from cross-sectional imaging will likely play an increasingly important role in the diagnosis and monitoring of cancer progression; however, there is a need to further understand the molecular programs underlying these features. There is increasing recognition that there is no one mechanism for the development and progression of cancer and that one malignant neoplastic phenotype can result from multiple alternative pathways (8-13). Gene dose is one mechanism that can lead to such transformations. We hypothesized that the classification of GBM tumors on the basis of clinical imaging features may lead to identification of genes in which transcription varied according to changes in gene dose and the number of copies of a gene within each cell (nucleus). By linking MR imaging features, mRNA measurements, and CNV measurements through independently evaluated correlations, it is possible to identify not only unique molecular mRNA and DNA subclasses of GBM on the basis of these imaging features but also MR phenotypes that may be due in part to changes in gene dose. 
The purpose of this study was to perform a multilevel radiogenomics study to elucidate the GBM MR imaging radiogenomic signatures resulting from changes in mRNA expression and DNA CNV.

\section{Materials and Methods \\ Imaging and Genomic Profiling}

This retrospective study used data generated from previously published institutional review board-approved Health Insurance Portability and Accountability Act-compliant studies that involved frozen samples from patients with brain tumors and preoperative MR images $(14,15)$. Tl-weighted pre- and postcontrast (Magnevist; Schering, Berlin, Germany), T2weighted, and fast low- angle shot MR imaging sequences were performed with a 1.5-T MR unit (Signa Echospeed; GE Medical Systems, Milwaukee, Wis) and were used to image the brains of 23 patients with histologically confirmed grade IV GBM according to the World Health Organization classification (16). The average age of male patients was 61 years (range, 20-79 years). The average age of female patients was 68 years (range, 55-79 years). The mean age of all patients was 62 years; there was no significant difference in patient age between groups $(P=.38)$. There were 18 male patients and five female patients $(P<.05)$. The image traits evaluated in this study were infiltrative versus edematous T2 abnormality, degree of contrast enhancement, necrosis, subventricular zone (SVZ) involvement, mass effect, and contrast-to-necrosis ratio, and they were evaluated by two experts (M.D.K., M.D.) in consensus according to predefined imaging trait definitions and exemplar images (Fig 1) that have been shown to be predictive of overall survival in patients with GBM and representative of underlying genetic signatures (1).

Imaging traits were defined according to the following features (Fig 1): (a) infiltrative versus edematous T2 abnormality, (b) degree of contrast enhancement, (c) necrosis, (d) SVZ involvement, (e) mass effect, and (f) contrast-to-necrosis ratio.

The infiltrative versus edematous $\mathrm{T} 2$ abnormality trait was defined in terms of three categories. The first is an edematous pattern in which there is a classic vasogenic edema pattern of the unenhanced portion of the tumor, where the $\mathrm{T} 2$ abnormality has a classic pseudopod appearance and a generally uniform T2 hyperintense signal. The second is a minimal edema pattern defined as the absence of any substantial vasogenic edema or infiltrative appearance. The third is an infiltrative pattern defined as a diffuse expansile T2 abnormality beyond the solid enhancing portion of the tumor that was slightly hyperintense compared to the normal brain and did not have the classic appearance of vasogenic edema. The binary version combined the edematous and minimal edema features into one category.

The degree of contrast enhancement was defined in terms of three categories: that in which it was $5 \%$ or less of the entire tumor volume, that in which it was more than $5 \%$ but less than or equal to $25 \%$ of the entire tumor volume, and that in which it was more than $25 \%$ of the entire tumor volume. The binary version consisted of two categories: contrast enhancement of $5 \%$ or less of the entire tumor volume and contrast enhancement of more than $5 \%$ of the entire tumor volume. 
The degree of necrosis was classified according to three categories: that in which there was absence of the central unenhanced region surrounded by a contrast-enhanced rim, that in which there was partial absence of a central nonenhancing region surrounded by a contrastenhanced rim, and that in which there was presence of a central unenhanced region surrounded by a contrast-enhanced rim. The binary version combined the second and third features into one category.

SVZ was classified according to one of two categories: that in which the contrast-enhanced region did not touch or involve the subependymal zone of the ventricles and that in which the contrast-enhanced region did touch or involve the subependymal zone of the ventricles.

Mass effect was classified in terms of three categories: the first was no or mild mass effect, in which there was no mass effect or a mild mass effect causing adjacent sulcal effacement or slight compression of adjacent structures but no gross alteration of shape. The second was moderate mass effect, in which there was compression of adjacent structures great enough to cause some alteration or distortion of the normal shape or shift of midline structures. The third was severe mass effect, in which there was compression of adjacent structures great enough to cause substantial distortion of the normal shape and midline shift. For the binary version of the trait, we combined the severe and moderate groups into a single category.

Contrast-to-necrosis ratio was classified in terms of three categories: A ratio of less than 1 indicated contrast-enhanced volume was significantly less than necrosis volume. A ratio of 1 indicated contrast-enhanced volume was approximately equal to the necrosis volume. A ratio of more than 1 indicated contrast-enhanced volume was greater than necrosis volume. The binary version combined the first two features into one category.

Global gene expression and genomic DNA measurements from the matched surgical GBM specimens with use of research identifiers were performed in these patients, as previously described (14). Briefly, nucleic acid extraction and hybridization were performed on custom complementary DNA arrays containing 41421 elements (corresponding to 27290 UniGene identifiers) for mRNA and DNA analysis from regions of histologically confirmed nonnecrotic tumor regions by using standard methods; the $\log 2$ mean normalized microarray (14) and array comparative genomic hybridization (15) profiles were subsequently downloaded from the Stanford Microarray Database for analysis (http://smd.stanford.edu/) for 54 partially overlapping patients. Adequate mRNA and DNA were not available for extraction in every patient; therefore, matching mRNA and DNA samples were not available for every patient (Fig E1 [online]). The data measurements were performed in the same laboratory and are available for download from the Stanford Microarray Database. Probes that did not have a gene annotation were removed. Probes that matched multiple genes were split into individual entries. 'Redundant' gene entries were collapsed by keeping the genes with the largest 1-norm. Gene and chromosome annotations were obtained from SOURCE (http://source.stanford.edu) (17) and RefSeq (http://ncbi.nlm.nih.gov/RefSeq/) by using unique gene Entrez identifiers (www.ncbi.nlm.nih.gov/sites/entrez/). 


\section{Data Analyses}

Approach.—-Two data analysis approaches, a global gene set enrichment approach and a more targeted individual gene-based approach, were implemented (Fig 2). The former focuses on identification of associations between predefined gene sets or pathways and imaging features, while the latter is used to assess individual gene loci for associations with imaging features, thereby providing a more specific detailed association map. The correlations between imaging features and genomic profiling measurements were assessed at multiple levels and combinations (eg, MR imaging and mRNA, MR imaging and CNV, and MR imaging, mRNA, and CNV). Each analysis was performed independent of any other. For each of the correlations with MR imaging, MR features were used to define different patient subgroups.

Radiogenomic gene set enrichment patterns.—Gene set enrichment refers to the general class of bioinformatic approaches used to identify significant changes in biologic pathways given a significant change in the expression of a subset of genes. We used gene set enrichment analysis (GSEA) (18) to obtain pathway level association maps with MR imaging features. Binary classification of the imaging traits was used to specify six different groups of traits to evaluate pathways or motifs associated with each of the signatures. Two different types of gene sets were used for analysis: the BioCarta Pathways (www.biocarta.com) and the GSEA literature-curated set of gene signatures. As with the mRNA profiling analysis, GSEA was used with binary classification of the six MR imaging traits for enrichment of pathways in the BioCarta collection and experimentally derived curated gene sets found in the literature for correlation with CNV.

For GSEA, two class comparison classifications were used that required conversion of the imaging trait read matrix into a binary matrix. Image trait scores were converted to ordinal integer scales and binary scales on the basis of the individual histograms for each trait across all of the samples, most of which approximated bimodal distributions. The binary cutoff criterion was determined independently for each trait to obtain as equally sized groups (or bins) as possible to avoid biasing the statistical tests by creating a small group with an artificially low variance. Prior to conversion into a binary matrix, the values of traits that could not be assessed were filled with the mean values of the traits across all of the other samples. The binary trait cutoff values were $2,2,1,1,1$, and 0 for contrast, necrosis, contrast-to-necrosis ratio, SVZ involvement, mass effect, and infiltrative versus edematous T2 abnormality, respectively. The set of imaging traits remained linearly independent before and after conversion to the binary classification scheme. Pearson correlation coefficient calculations were performed by using integer-scale trait score values.

Gene set enrichment was performed in the subset of patients in whom imaging data, mRNA profiling, and DNA copy number profiling were obtained. The final data sets included 14 561 and 14544 unique elements for mRNA and DNA arrays, respectively, across 16 patients. This constituted the number of patients for whom mRNA profiles, CNV profiles, and imaging features were available (Fig E1 [online]). Bioinformatic analysis was performed to identify enriched biologic pathways in each data set independently with the binary classification of the MR imaging trait scores. GSEA (18) is widely used to analyze 
and interpret expression profiling in terms of predefined pathway sets of interest. The BioCarta and genetic and chemical perturbation gene sets from the curated gene sets (www.broadinstitute.org/gsea/msigdb) were used from GSEA (18). A nominal $P$ value of less than .05 was used to determine inclusion of a particular pathway.

Radiogenomic associations. - The mRNA and CNV data sets were available in 45 of the patients in the original publications (the shared set of patients between mRNA and $\mathrm{CNV}$ data sets); thus, correlation between mRNA and CNV was performed in all of these patients. Subsequent analysis and correlation with imaging features was performed in the subset of 23 patients for whom MR images were available. To decrease effects from background gene expression noise, the mRNA and CNV data sets were filtered by keeping the genes with the top 35\% of variance (13 887 and 13857 for mRNA and CNV, respectively) and then selecting only those genes that were shared between the two data sets (3650 gene loci). An unpaired two-tailed $t$ test with 20000 permutations without replacement to correct for multiple hypothesis testing was performed between the two data sets. The false discovery rate was calculated with Matlab software (MathWorks, Natick, Mass), as described by Storey (19). The set of genes with significant positive Pearson correlation coefficients (ie, concordantly changing gene expression and copy number variation) was determined with filtering based on a corrected $P$ value of less than .01 and a false discovery rate of less than .01. Finally, analysis of variance was performed on the set of imaging features by using MR imaging trait values to define the different comparison groups for the set of correlated mRNA-CNV genes $(P<.05)$.

GSEA was downloaded from the Bioconductor software repository (www.bioconductor.org). Calculations and simulations were performed with R (cran.r-project.org) and Matlab (Math Works) software.

\section{Results} MR Imaging

mRNA pathway enrichment correlations._-A total of 30 BioCarta gene sets and 66 curated pathway gene sets were significantly correlated with the six MR imaging traits. The BioCarta Cytokine Pathway was significantly associated with presence of necrosis at imaging and a low contrast-to-necrosis ratio. The absence of mass effect was associated with the interleukin 3, wingless type integration site family, integrin, and transforming growth factor $\beta$ pathways (Fig 3). High contrast enhancement was associated with the largest number of BioCarta pathways $(n=15)$, which included epidermal growth factor, cAMP responsive element binding protein, mitogen-activated protein kinase, tolllike receptor family, and insulinlike growth factor pathways, among others (Fig 3).

Overall, results from the experimentally derived gene signatures highlighted complementary biologic pathways and mechanisms that would be expected to be altered in patients with cancer, such as hypoxia, changes in copy number, and activation of different signaling pathways related to angiogenic processes. Notably, a lack of SVZ involvement was associated with downregulation of a glioma stem cell signature, which is consistent with the hypothesis that stem cells residing in the SVZ may progress to GBM $(20,21)$. Conversely, 
SVZ involvement was associated with decreased hypoxia signatures, changes in copy number (increased with colon cancer but decreased with a melanoma copy number signature), and increased tumor vascularity and invasion. Gene expression signatures involving copy number changes appeared to be enriched in traits indicating changes in contrast enhancement. Increased edema was associated with hypoxia and increased metastasis signatures.

CNV pathway enrichment correlations. - Thirty-two BioCarta gene sets and 53 curated pathway gene sets were significantly correlated with the six MR imaging traits. A lack of SVZ involvement was associated with the BioCarta Cytokine, Inflammation, and p53 hypoxia pathways, while SVZ involvement was associated with vascular endothelial growth factor, extracellular matrix, and chemokine (C-C motif) receptor 5 pathways (Fig 4). A lack of necrosis was associated with the cell cycle and related signaling pathways (Fig 4). Enrichment in BioCarta pathways for increased contrast-to-necrosis ratio overlapped with pathways identified with decreased necrosis and a lack of SVZ involvement. Overall, the edematous trait demonstrated the greatest enrichment in the BioCarta pathways. The contrast-related traits demonstrated the greatest enrichment in the experimentally derived gene signatures.

\section{Comparison of MR Imaging and mRNA Correlation with MR Imaging and CNV Correlation}

A comparison of pathway enrichment with Biocarta pathways and the experimentally derived gene sets revealed, as would be expected, a smaller number of concordant changes between DNA copy number and gene expression at the agglomerated pathway level. However, notable correlations between gene dose and mRNA expression included decreased necrosis and a high contrast-to-necrosis ratio, both of which were associated with hypoxiarelated signatures in the mRNA and array comparative genomic hybridization results. Such observations warrant the need to analyze correlations on a gene-by-gene basis rather than on a more global pathway level to achieve a more insightful integrated analysis.

\section{MR imaging, mRNA, and CNV Integration}

Integration of MR imaging, mRNA, and CNV data resulted in identification of individual genes and loci with correlated mRNA and CNV changes that are also significantly associated with particular imaging features. A total of 376 genes had concordant correlated mRNA and CNV changes. From these genes, a total of 34 unique genes or loci were identified as being significantly correlated with at least one of the six imaging features (Table, Fig 4). Most of the imaging features were associated with five or six different genes, with the exception of the SVZ involvement trait, which was associated with 13 different genes. Functional classes of identified genes include oncogenes, transcription factors, metabolic enzymes, signaling molecules, and receptor proteins, among others. Many of these genes have been associated with or are specific to neuronal functions. In addition, almost one-third of the genes localize to chromosome 1, particularly the $p$ arm. 


\section{Discussion}

The radiogenomic association map in the Table highlights the genes identified to have concordant mRNA expression and gene dose changes, as well as significant correlations with the different MR imaging features analyzed in this cohort of patients. Some of the genes have already been shown to be biomarkers for GBM, and many of the other genes have shown clear associations with tumorigenesis in different cell and tissue types. Speculation about all of the potential associations and mechanist causes is clearly not warranted; however, we note a few genes of interest associated with different imaging features.

The association of contrast enhancement with $L T B P 1$ suggests that it may serve as an imaging biomarker for more aggressive phenotypes of GBM. LTBP1 modulates the secretion and processing of transforming growth factor- $\beta$, a cytokine that has been targeted in a number of malignancies (22). In particular, transforming growth factor- $\beta$ has been associated with more malignant gliomas, and overexpression of $L T B P 1$ has been associated with increased grade of malignancy in gliomas (23). The associations identified in this study suggest that these findings may also extend to GBM. Increased expression of RUNX3 has been described as having inhibitory effects on cell migration and invasion, and epigenetic silencing of RUNX3 has been previously observed in patients with $\operatorname{GBM}(24,25)$.

Since contrast enhancement and necrosis are not mutually exclusive features in patients with GBM, the ratio of the two characteristics results in an independent imaging feature. The epithelial mesenchymal transition has received increasing attention relatively recently, as the changes are viewed to be important in conferring the ability to metastasize to cancer cells. To our knowledge, no researchers have identified associations between GBM and $K L K 3$, a member of a family of serine proteases; however, $K L K 3$ has been implicated as an associated gene in other malignancies, such as prostate cancer, and it is thought to have a potential role as a general biomarker of malignancy $(26,27)$.

SVZ involvement or lack thereof was associated with the largest number of individual genes (13) in the analysis of this patient cohort. CHI3L1, a member of the chitinase hydrolytic enzyme family involved in tissue remodeling processes, has been reported to possibly be a better predictor of overall survival than $M G M T$ status in patients with GBM (28). RAP2A is a member of the G-protein Ras oncogene family, and decreased expression of $R A P 2 A$ has been observed to promote in vitro migration and invasion of glioma cells (29). Alterations in expression of the HJURP have been associated with chromosomal changes in multiple tumors (30-32) and have been identified as a negative predictor of outcome in patients with high-grade gliomas (33). TYMS is a metabolic enzyme that has been targeted with raltitrexed to inhibit DNA synthesis (34). Recent analyses have actually postulated that raltitrexed may block cell proliferation in glioblastoma cell lines (35). LMO3 also belongs to an oncogene family, and altered expression with epigenetic-related changes has been described in patients with lung cancer and those with neuroblastoma $(36,37)$.

The presence or absence of mass effect and the infiltrative versus edematous appearance of tumors were also associated with genes with causal roles identified in different 
malignancies. Some of these genes may have utility in prognostic and treatment target identification. SPAG5 has been identified as a regulator of mitotic spindles and has been proposed as a biomarker in lung and breast cancer $(38,39)$. ITIH2 is a member of the plasma protease inhibitor family and has been observed to be downregulated in patients with breast, lung, or colon cancer (40). FOXP1 has been identified as a prognostic predictor of hepatocellular carcinoma and non-small cell lung cancer (41,42). PIK3IP1, a negative regulator of phos-phoinositide-3-kinase, which is an integral component of the mechanistic target of rapamycin ( $m \mathrm{TOR}$ )apoptosis signaling pathway, has been shown to suppress the development of hepatocellular carcinoma (43). Epigenetic changes of RASSF2 have been described in patients with different types of cancer, with correlations with clinical markers and potential applications as a cancer biomarker (44-48). CLDN10, a member of the claudin membrane proteins that mediate intercellular tight junctions, has been implicated in numerous malignancies and has shown potential as a prognostic biomarker in patients with cancer (49).

In summary, this study has shown that multilevel radiogenomic association maps can be created. The construction of such a map by using MR imaging, mRNA, and CNV profiles in patients with GBM on both a pathway level and a gene level has led to insight into some of the gene dose-related features that are associated with the macroscopic appearance of the tumor. A practical limitation in performing such a study is the ability to obtain independent cohorts of patients with appropriate imaging studies and adequate tissue samples to perform multilevel high-throughput (eg, genomic, transcrip-tomic, proteomic, metabolomic, etc) measurements. While public repositories, such as those supported by The Cancer Genome Atlas (http://cancergenome.nih.gov/) continue to grow and expand, it is important to procure, develop, and evaluate additional data sets to ensure depth and breadth in the data sets available for analysis.

As with most studies that require integration of large disparate data measurements, a limitation of this study was the sample size. Because we performed the different arms of the analysis independently while also accounting for multiple hypotheses testing, the number of strongly linked correlates was expected to be small. However, it is necessary to characterize and identify the correlative features and genes in smaller studies to help drive and direct future larger studies. Ultimately, these results support the need for and utility of deeper characterization of the interpretation of imaging features by using high-throughput measurements. Such approaches, as shown, highlight the potential power of radiogenomic strategies to glean further insight into the molecular biology of individual tumors while leveraging existing imaging modalities. By scaling the study to include additional dimensions of biologic data, such as array comparative genomic hybridization, as shown here, one may begin to gain causative insight into individual imaging phenotypes. Future studies involving larger cohorts of patients are likely to result in identification of gene signatures for other imaging features.

Imaging trait predictors that are correlated with one another in array comparative genomic hybridization and expression profiling may be indicative of underlying biologic relationships between gene transcriptional activity and gene dose. Identification of intersections of independent correlations with imaging features is one approach to distilling complex data 
sets into manageable pieces and drilling down to potentially causal relationships. Imaging features, including necrosis, contrast enhancement, and spatial localization of brain tumors, were found to have significant alterations in mRNA expression and gene dose levels in some of the genes implicated in the pathogenesis of GBM. While further characterization of these imaging features reflecting different enhancement patterns and tumor localization is needed through more extensive genomic profiling and in larger patient cohorts, this initial MR imaging, mRNA, and CNV GBM map is a step toward the further elucidation of radiogenomic features of GBM.

\title{
Supplementary Material
}

Refer to Web version on PubMed Central for supplementary material.

\author{
Abbreviations: \\ CNV copy number variation \\ GBM glioblastoma multiforme \\ GSEA gene set enrichment analysis \\ mRNA messenger RNA \\ SVZ subventricular zone
}

\section{References}

1. Diehn M, Nardini C, Wang DS, et al. Identification of noninvasive imaging surrogates for brain tumor gene-expression modules. Proc Natl Acad Sci US A 2008;105(13): 5213-5218.

2. Kuo MD, Gollub J, Sirlin CB, Ooi C, Chen X. Radiogenomic analysis to identify imaging phenotypes associated with drug response gene expression programs in hepatocellular carcinoma. $\mathbf{J}$ Vasc Interv Radiol 2007;18(7): 821-831. [PubMed: 17609439]

3. Rutman AM, Kuo MD. Radiogenomics: creating a link between molecular diagnostics and diagnostic imaging. Eur J Radiol 2009; 70(2):232-241. [PubMed: 19303233]

4. Barajas RF Jr, Phillips JJ, Parvataneni R, et al. Regional variation in histopathologic features of tumor specimens from treatment-naive glioblastoma correlates with anatomic and physiologic MR imaging. Neuro-oncol 2012; 14(7):942-954. [PubMed: 22711606]

5. Carrillo JA, Lai A, Nghiemphu PL, et al. Relationship between tumor enhancement, edema, IDH1 mutational status, MGMT promoter methylation, and survival in glioblastoma. AJNR Am J Neuroradiol 2012;33(7): 1349-1355. [PubMed: 22322613]

6. Gutman DA, Cooper LA, Hwang SN, et al. MR imaging predictors of molecular profile and survival: multi-institutional study of the TCGA glioblastoma data set. Radiology 2013; 267(2):560569. [PubMed: 23392431]

7. Zinn PO, Mahajan B, Sathyan P, et al. Radiogenomic mapping of edema/cellular invasion MRIphenotypes in glioblastoma multiforme. PLoS ONE 2011;6(10):e25451. [PubMed: 21998659]

8. Chamberlain M Evolving strategies: future treatment of glioblastoma. Expert Rev Neu-rother 2011;11(4):519-532.

9. Hoang-Xuan K, He J, Huguet S, et al. Molecular heterogeneity of oligodendrogliomas suggests alternative pathways in tumor progression. Neurology 2001;57(7):1278-1281. [PubMed: 11591848]

10. Lamont KR, Tindall DJ. Minireview: alternative activation pathways for the androgen receptor in prostate cancer. Mol Endocrinol 2011;25(6):897-907. [PubMed: 21436259] 
11. McCormick JJ, Maher VM. Malignant transformation of human skin fibroblasts by two alternative pathways. Adv Exp Med Biol 2011; 720:191-207. [PubMed: 21901629]

12. Sathornsumetee $S$ Therapeutic strategies to target multiple kinases in glioblastoma. Anticancer Agents Med Chem 2011 ;11 (8): 700-711. [PubMed: 21707500]

13. Smith G, Carey FA, Beattie J, et al. Mutations in APC, Kirsten-ras, and p53: alternative genetic pathways to colorectal cancer. Proc Natl Acad Sci U S A 2002;99(14):9433-9438. [PubMed: 12093899]

14. Bredel M, Bredel C, Juric D, et al. Functional network analysis reveals extended gliomagenesis pathway maps and three novel MYC-interacting genes in human gliomas. Cancer Res 2005;65(19):8679-8689. [PubMed: 16204036]

15. Bredel M, Bredel C, Juric D, et al. Highresolution genome-wide mapping of genetic alterations in human glial brain tumors. Cancer Res 2005;65(10):4088-4096. [PubMed: 15899798]

16. Kleihues P, Louis DN, Scheithauer BW, et al. The WHO classification of tumors of the nervous system. J Neuropathol Exp Neurol 2002;61(3):215-225; discussion 226-229. [PubMed: 11895036]

17. Diehn M, Sherlock G, Binkley G, et al. SOURCE: a unified genomic resource of functional annotations, ontologies, and gene expression data. Nucleic Acids Res 2003;31(1): 219-223. [PubMed: 12519986]

18. Subramanian A, Tamayo P, Mootha VK, et al. Gene set enrichment analysis: a knowledge-based approach for interpreting genome-wide expression profiles. Proc Natl Acad Sci U S A 2005;102(43):15545-15550. [PubMed: 16199517]

19. Storey J A direct approach to false discovery rates. J R Stat Soc Series B Stat Methodol 2002;64(3):479-498.

20. Glantz M, Kesari S, Recht L, Fleischhack G, Van Horn A. Understanding the origins of gliomas and developing novel therapies: cerebrospinal fluid and subventricular zone interplay. Semin Oncol 2009;36(4 Suppl 2): S17-S24. [PubMed: 19660679]

21. Lim DA, Cha S, Mayo MC, et al. Relationship of glioblastoma multiforme to neural stem cell regions predicts invasive and multifocal tumor phenotype. Neuro-oncol 2007;9(4): 424-429. [PubMed: 17622647]

22. Oklü R, Hesketh R. The latent transforming growth factor beta binding protein (LTBP) family. Biochem J 2000;352(Pt 3):601-610. [PubMed: 11104663]

23. Tritschler I, Gramatzki D, Capper D, et al. Modulation of TGF-beta activity by latent TGF-betabinding protein 1 in human malignant glioma cells. Int J Cancer 2009;125(3): 530-540. [PubMed: 19431147]

24. Mei PJ, Bai J, Liu H, et al. RUNX3 expression is lost in glioma and its restoration causes drastic suppression of tumor invasion and migration. J Cancer Res Clin Oncol 2011; 137(12):1823-1830. [PubMed: 21922326]

25. Mueller W, Nutt CL, Ehrich M, et al. Down-regulation of RUNX3 and TES by hypermeth-ylation in glioblastoma. Oncogene 2007; 26(4):583-593. [PubMed: 16909125]

26. Emami N, Diamandis EP. Utility of kallikrein-related peptidases (KLKs) as cancer biomarkers. Clin Chem 2008;54(10):1600-1607. [PubMed: 18687738]

27. Lawrence MG, Veveris-Lowe TL, Whitbread AK, Nicol DL, Clements JA. Epithelial-mesenchymal transition in prostate cancer and the potential role of kallikrein serine proteases. Cells Tissues Organs 2007;185(1-3):111-115. [PubMed: 17587816]

28. Salvati M, Pichierri A, Piccirilli M, et al. Extent of tumor removal and molecular markers in cerebral glioblastoma: a combined prognostic factors study in a surgical series of 105 patients. $\mathbf{J}$ Neurosurg 2012; 117(2): 204-211. [PubMed: 22655594]

29. Choudhury Y, Tay FC, Lam DH, et al. Attenuated adenosine-to-inosine editing of microRNA-376a* promotes invasiveness of glioblastoma cells. J Clin Invest 2012;122(11): 40594076. [PubMed: 23093778]

30. Hu Z, Huang G, Sadanandam A, et al. The expression level of HJURP has an independent prognostic impact and predicts the sensitivity to radiotherapy in breast cancer. Breast Cancer Res 2010;12(2):R18. [PubMed: 20211017] 
31. Kato T, Sato N, Hayama S, et al. Activation of Holliday junction recognizing protein involved in the chromosomal stability and immortality of cancer cells. Cancer Res 2007;67(18): 8544-8553. [PubMed: 17823411]

32. Valente V, Teixeira SA, Neder L, et al. Selection of suitable housekeeping genes for expression analysis in glioblastoma using quantitative RT-PCR. BMC Mol Biol 2009; 10:17. [PubMed: 19257903]

33. de Tayrac M, Aubry M, Saïkali S, et al. A 4-gene signature associated with clinical outcome in high-grade gliomas. Clin Cancer Res 2011;17(2):317-327. [PubMed: 21224364]

34. Touroutoglou N, Pazdur R. Thymidylate synthase inhibitors. Clin Cancer Res 1996;2(2): 227-243. [PubMed: 9816165]

35. Mpindi JP, Sara H, Haapa-Paananen S, et al. GTI: a novel algorithm for identifying outlier gene expression profiles from integrated microarray datasets. PLoS ONE 2011;6(2): e17259. [PubMed: 21365010]

36. Aoyama M, Ozaki T, Inuzuka H, et al. LMO3 interacts with neuronal transcription factor, HEN2, and acts as an oncogene in neuroblastoma. Cancer Res 2005;65(11):4587-4597. [PubMed: 15930276]

37. Kwon YJ, Lee SJ, Koh JS, et al. Genome-wide analysis of DNA methylation and the gene expression change in lung cancer. J Thorac Oncol 2012;7(1):20-33. [PubMed: 22011669]

38. Buechler S Low expression of a few genes indicates good prognosis in estrogen receptor positive breast cancer. BMC Cancer 2009;9:243. [PubMed: 19619298]

39. Välk K, Vooder T, Kolde R, et al. Gene expression profiles of non-small cell lung cancer: survival prediction and new biomarkers. Oncology 2010;79(3-4):283-292. [PubMed: 21412013]

40. Hamm A, Veeck J, Bektas N, et al. Frequent expression loss of inter-alpha-trypsin inhibitor heavy chain (ITIH) genes in multiple human solid tumors: a systematic expression analysis. BMC Cancer 2008;8:25. [PubMed: 18226209]

41. Feng J, Zhang X, Zhu H, Wang X, Ni S, Huang J. High expression of FoxP1 is associated with improved survival in patients with non-small cell lung cancer. Am J Clin Pathol 2012;138(2):230 235. [PubMed: 22904134]

42. Zhang Y, Zhang S, Wang X, et al. Prognostic significance of FOXP1 as an oncogene in hepatocellular carcinoma. J Clin Pathol 2012; 65(6):528-533. [PubMed: 22422806]

43. He X, Zhu Z, Johnson C, et al. PIK3IP1, a negative regulator of PI3K, suppresses the development of hepatocellular carcinoma. Cancer Res 2008;68(14):5591-5598. [PubMed: 18632611]

44. Hiraki M, Kitajima Y, Koga Y, et al. Aberrant gene methylation is a biomarker for the detection of cancer cells in peritoneal wash samples from advanced gastric cancer patients. Ann Surg Oncol 2011;18(10):3013-3019. [PubMed: 21409489]

45. Imai T, Toyota M, Suzuki H, et al. Epigenetic inactivation of RASSF2 in oral squamous cell carcinoma. Cancer Sci 2008;99(5):958-966. [PubMed: 18294275]

46. Kaira K, Sunaga N, Tomizawa Y, et al. Epigenetic inactivation of the RAS-effector gene RASSF2 in lung cancers. Int J Oncol 2007; 31(1):169-173. [PubMed: 17549418]

47. Ren J, He W, Zhang R, et al. RASSF2A promoter methylation in hepatitis B virus-related hepatocellular carcinogenesis and its correlation with elevated serum alpha-fetoprotein level. $\mathrm{J}$ Huazhong Univ Sci Technolog Med Sci 2009;29(3):309-312. [PubMed: 19513612]

48. Zhao L, Cui Q, Lu Z, Chen J. Aberrant methylation of RASSF2A in human pancreatic ductal adenocarcinoma and its relation to clinicopathologic features. Pancreas 2012;41(2):206-211 [PubMed: 21792082]

49. Ouban A, Ahmed AA. Claudins in human cancer: a review. Histol Histopathol 2010; 25(1):83-90. [PubMed: 19924644] 


\section{Advances in knowledge}

- Six MR imaging features showed correlation $(P<.05)$ with concordant variations in gene dose and messenger RNA (mRNA) expression $(P<.01$, false discovery rate $<0.01$ for DNA and mRNA correlation) of 34 genes and loci, the majority of which are localized to chromosome $1 \mathrm{p}$ and are oncogenes or have been described as involved in tumori-genic processes and cell adhesion metastatic processes.

- Many of these genes have been implicated in other malignancies but have not been implicated in glioblastoma multiforme (GBM); therefore, they represent targets to further evaluate as biomarkers in patients with GBM.

\section{Implication for Patient Care}

- An integrated mRNA and DNA radiogenomic association map for GBM presents a means for noninvasive assessment of genomic signatures in patients with GBM by using MR imaging.

- Given considerations of sample size, these findings should be tested in a larger data set. 

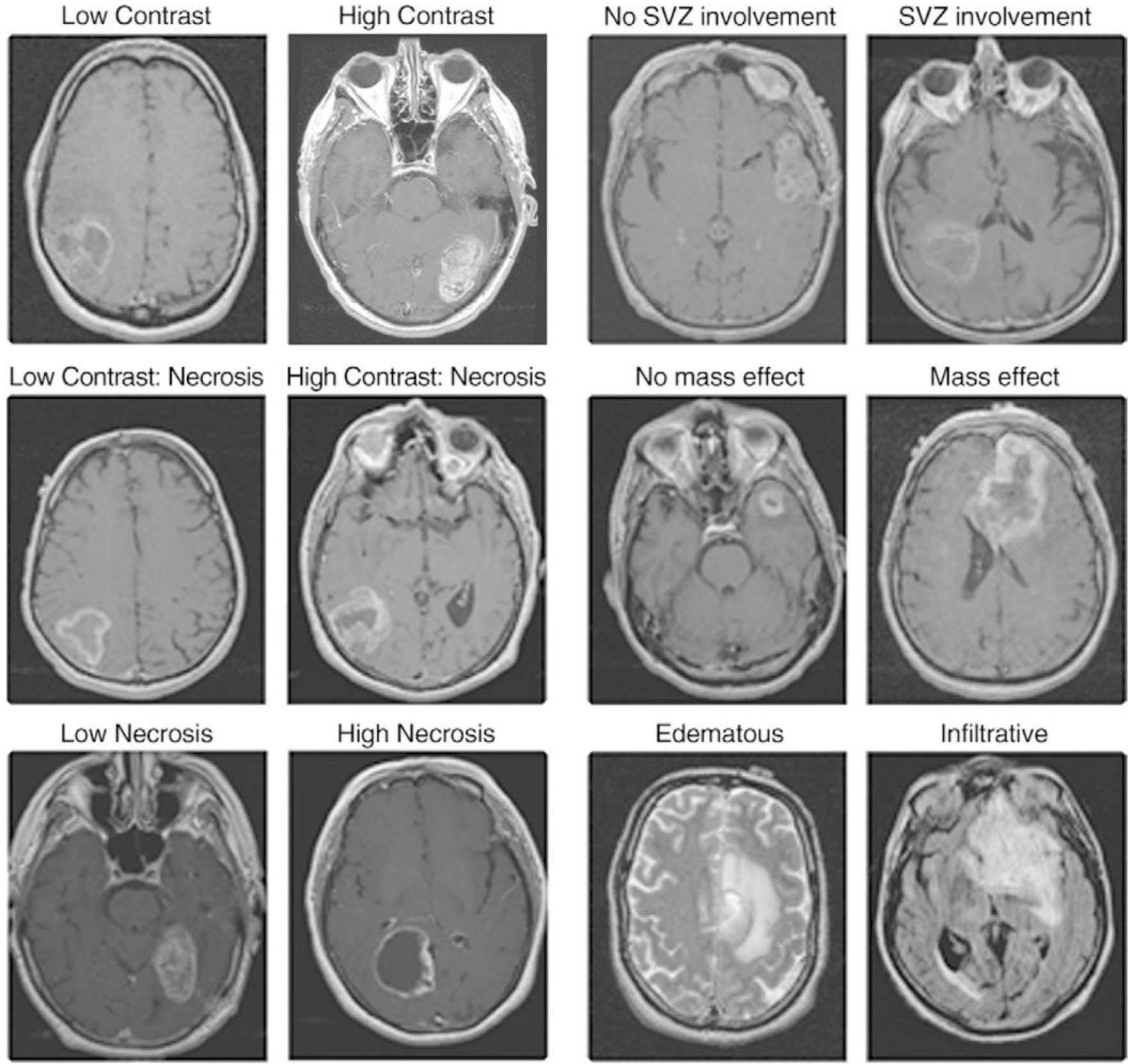

Figure 1:

Panel of exemplar images (binary version) of the MR imaging traits for the analyses in this article. 

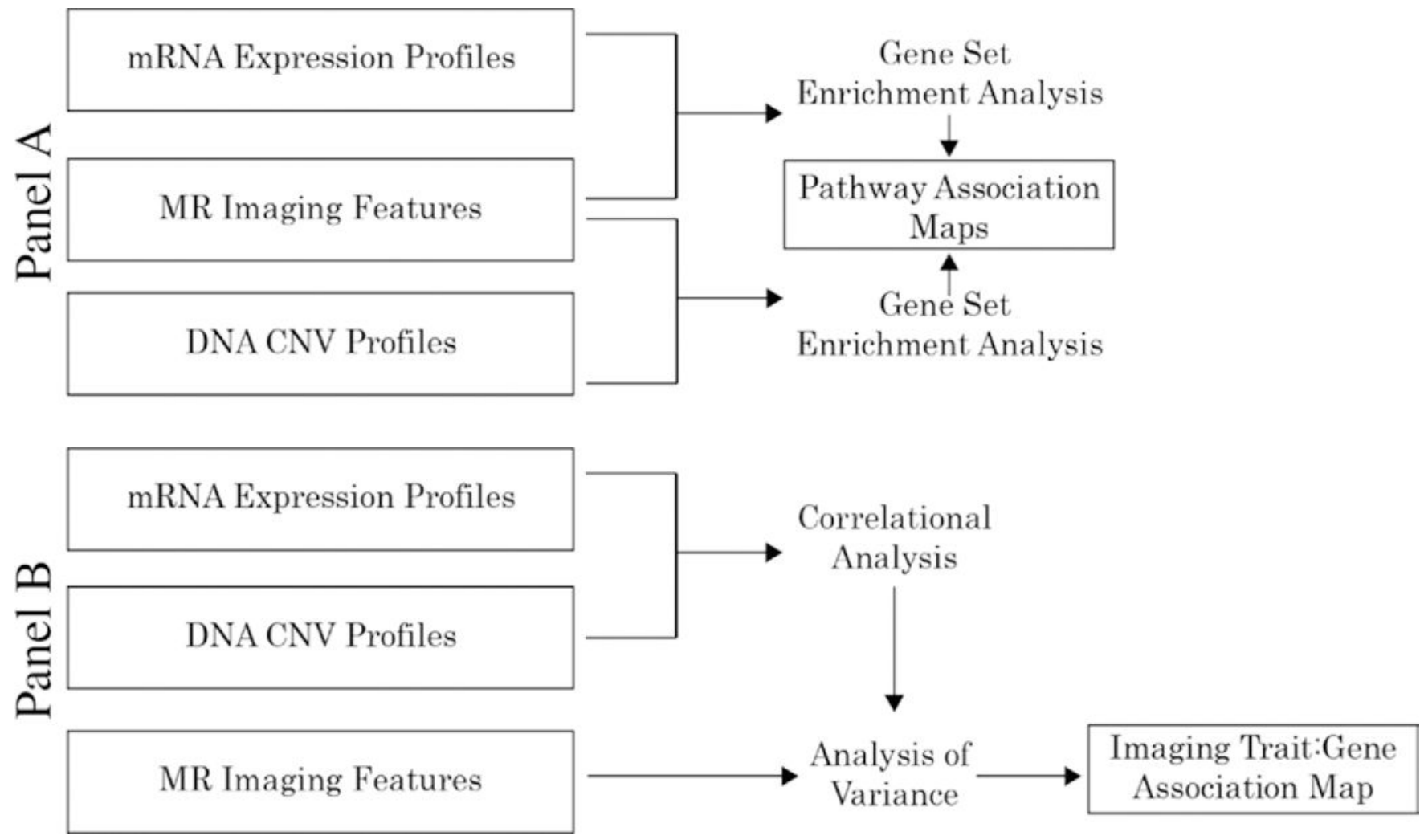

Figure 2:

Schematic outline of analysis approaches. Panel A delineates the global pathway association based approach implemented through GSEA. Associations between each genomic data set and imaging features were conducted independently and then analyzed with GSEA. The alternative integrative approach (Panel B) was used to identify concordant significant associations between the mRNA expression data set and the DNA CNV data set and then correlated with MR imaging features. The former approach yields a more global view of biologic processes associated with imaging features, whereas the latter enables definition of a more specific radiogenomic association map. 

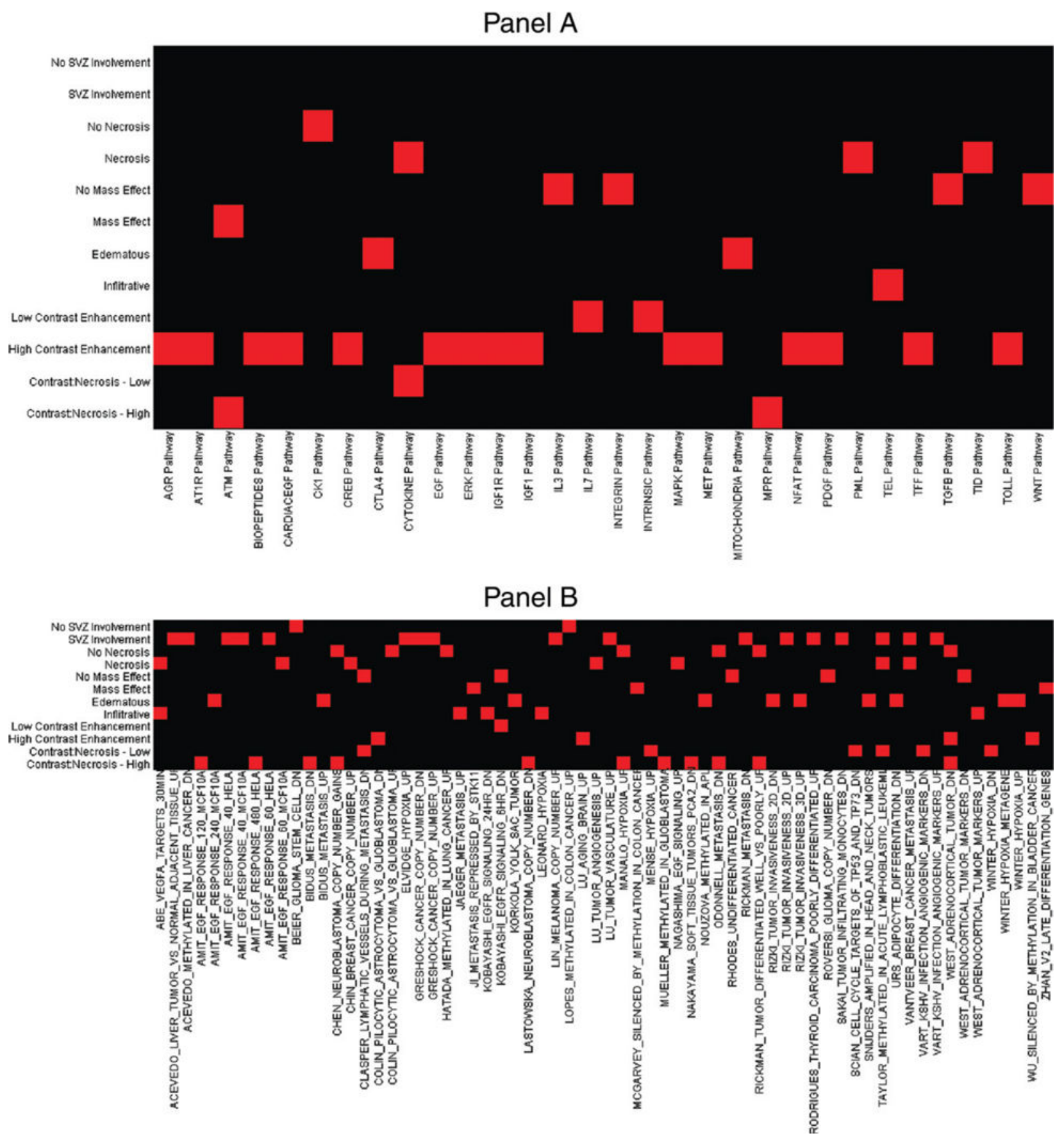

Figure 3:

GSEA of MR imaging trait classification with mRNA expression profiles. This summary figure highlights the different molecular pathways that are correlated with different imaging features (red boxes) on the basis of mRNA gene expression. Each pathway represents a different combination of genes. Panel A shows absence or presence for enrichment of BioCarta pathways with different imaging traits. Panel B shows absence or presence of enrichment of experimentally identified curated pathway signature sets with different imaging traits. 


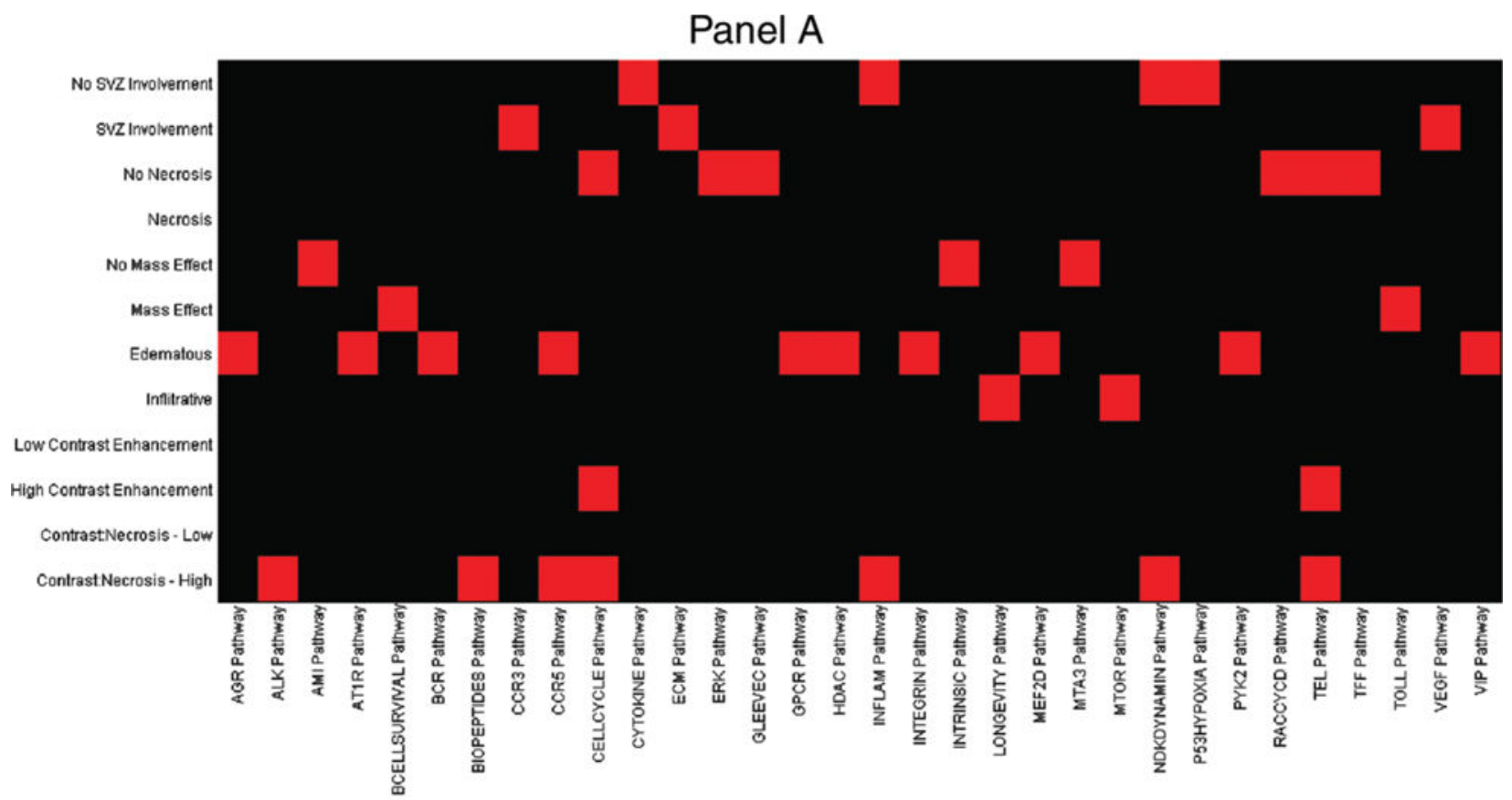

Panel B

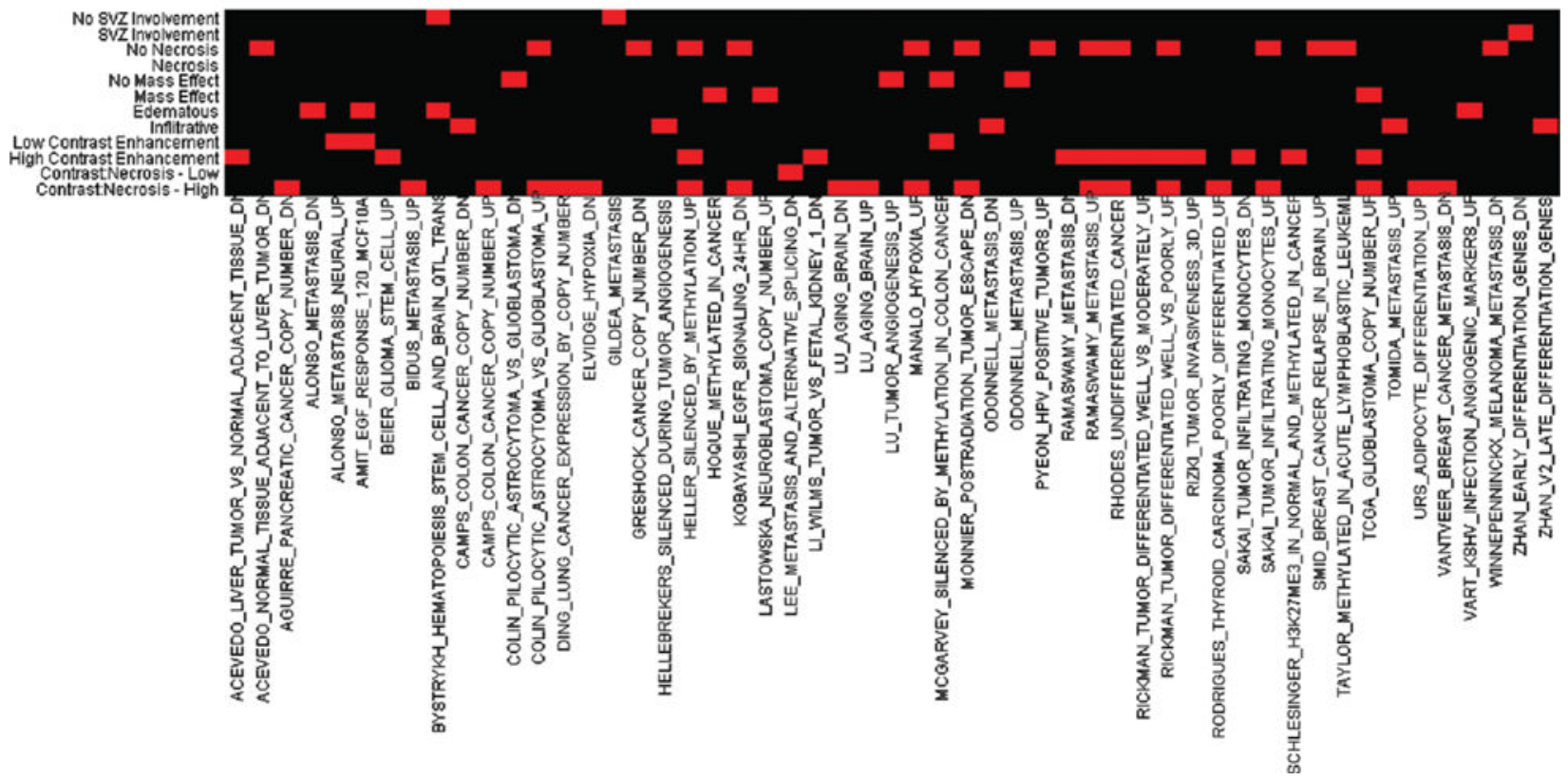

Figure 4:

GSEA analysis of MR imaging trait classification by DNA copy number profiles. This summary figure highlights the different molecular pathways that are correlated with different imaging features (red box) on the basis of DNA CNV. Each pathway represents a different combination of genes. Panel A shows absence or presence for enrichment of BioCarta pathways with different imaging traits. Panel B shows absence or presence of enrichment of experimentally identified curated pathway signature sets with different imaging traits. 
Table

GBM Radiogenomic Associations

\begin{tabular}{|c|c|c|c|c|c|}
\hline $\begin{array}{l}\text { Imaging Trait and } \\
\text { Gene Name }\end{array}$ & Entrez ID & Location & Gene Description & Association & $P$ Value \\
\hline \multicolumn{6}{|l|}{ Contrast } \\
\hline C1orf172 & 126695 & $1 \mathrm{p} 36.11$ & Chromosome 1 open reading frame 172 & Positive & .037 \\
\hline CAMSAP2 & 23271 & $1 \mathrm{q} 32.1$ & Calmodulin regulated spectrin-associated protein 1-like 1 & Positive & .008 \\
\hline KCNK3 & 3777 & $2 \mathrm{p} 23$ & Potassium channel, subfamily $\mathrm{K}$, member 3 & Positive & .001 \\
\hline LTBP1 & 4052 & p22-p21 & Latent transforming growth factor beta binding protein 1 & Positive & .019 \\
\hline \multicolumn{6}{|l|}{ Necrosis } \\
\hline ITGA5 & 3678 & $12 q 11-q 13$ & Integrin, alpha 5 (fibronectin receptor, alpha polypeptide) & Negative & .008 \\
\hline$R U N X 3$ & 864 & $1 \mathrm{p} 36$ & Runt-related transcription factor 3 & Negative & .003 \\
\hline \multicolumn{6}{|c|}{ Contrast-to-necrosis ratio } \\
\hline$K L K 3$ & 354 & $19 \mathrm{q} 13.41$ & Kallikrein-related peptidase 3 & Positive & .032 \\
\hline$I L 7 R$ & 3575 & $5 \mathrm{p} 13$ & Interleukin 7 receptor & Positive & .003 \\
\hline RBP4 & 5950 & $10 \mathrm{q} 23-\mathrm{q} 24$ & Retinol binding protein 4 , plasma & Positive & .004 \\
\hline RUNX3 & 864 & $1 \mathrm{p} 36$ & Runt-related transcription factor 3 & Positive & .003 \\
\hline MS4A1 & 931 & $11 \mathrm{q} 12-\mathrm{q} 13.1$ & Membrane-spanning 4-domains, subfamily A, member 1 & Positive & .035 \\
\hline \multicolumn{6}{|l|}{ SVZ } \\
\hline CHI3L1 & 1116 & $1 \mathrm{q} 32.1$ & Chitinase 3-like 1 (cartilage glycoprotein-39) & Negative & .025 \\
\hline ССDC8O & 151887 & $3 q 13.2$ & Coiled-coil domain containing 80 & Negative & .001 \\
\hline$E C M 2$ & 1842 & $9 \mathrm{q} 22.3$ & $\begin{array}{l}\text { Extracellular matrix protein 2, female organ and adipocyte } \\
\text { specific }\end{array}$ & Negative & .005 \\
\hline S1PR1 & 1901 & $1 \mathrm{p} 21$ & Sphingosine-1-phospate receptor 1 & Negative & .011 \\
\hline$U B Q L N 1$ & 29979 & $9 \mathrm{q} 22$ & Ubiquilin 1 & Positive & .015 \\
\hline$A P B A 1$ & 320 & $9 q 13-q 21$ & $\begin{array}{l}\text { Amyloid beta (A4) precursor protein-binding, family A, } \\
\text { member } 1\end{array}$ & Positive & .049 \\
\hline HJURP & 55355 & $2 \mathrm{q} 37.1$ & Holliday junction recognition protein & Positive & .038 \\
\hline LMO3 & 55885 & $12 \mathrm{p} 13$ & LIM domain only 3 (rhombot in-like 2) & Positive & .049 \\
\hline$R A P 2 A$ & 5911 & $13 q 34$ & RAP2A, member of RAS oncogene family & Positive & .032 \\
\hline$B F S P 1$ & 631 & $20 \mathrm{p} 12.1$ & Beaded filament structural protein 1, filensin & Positive & .021 \\
\hline TYMS & 7298 & 18p11.31-p11.21 & Thymidylate synthetase & Positive & .035 \\
\hline FAM83D & 81610 & 20 & Family with sequence similarity 83 , member D & Positive & .035 \\
\hline$M A E L$ & 84944 & $1 \mathrm{q} 24.1$ & Maelstrom spermatogenic transposon silencer & Negative & .015 \\
\hline \multicolumn{6}{|l|}{ Mass effect } \\
\hline CD52 & 1043 & $1 \mathrm{p} 36$ & CD52 molecule & Positive & .007 \\
\hline SPAG5 & 10615 & $17 \mathrm{q} 11.2$ & Sperm associated antigen 5 & Negative & .025 \\
\hline POC5 & 134359 & $5 q 13.3$ & POC5 centriolar protein & Negative & .041 \\
\hline$H E Y L$ & 26508 & $1 \mathrm{p} 34.3$ & Hairy/enhancer-of-split related with YRPW motif-like & Negative & .018 \\
\hline$I L 7 R$ & 3575 & $5 \mathrm{p} 13$ & Interleukin 7 receptor & Positive & .003 \\
\hline ITIH 2 & 3698 & $10 \mathrm{p} 14$ & Inter-alpha-trypsin inhibitor heavy chain 2 & Positive & .008 \\
\hline \multicolumn{6}{|c|}{ Infiltrative versus edematous * } \\
\hline PIK3IP1 & 113791 & $22 \mathrm{q} 12.2$ & Phosphoinositide-3-kinase interacting protein 1 & Negative & .018 \\
\hline
\end{tabular}




\begin{tabular}{lrllll}
\hline $\begin{array}{l}\text { Imaging Trait and } \\
\text { Gene Name }\end{array}$ & Entrez ID & Location & Gene Description & Association & $P$ Value \\
\hline FOXP1 & 27086 & $3 \mathrm{p} 14.1$ & Forkhead box P1 & Negative & .018 \\
APOB & 338 & $2 \mathrm{p} 24-\mathrm{p} 23$ & Apolipoprotein B & Negative & .025 \\
CLDN10 & 9071 & $13 \mathrm{q} 31-\mathrm{q} 34$ & Claudin 10 & Negative & .007 \\
NEXN & 91624 & $1 \mathrm{p} 31.1$ & Nexlin (F actin binding protein) & Negative & .005 \\
RASSF2 & 9770 & $20 \mathrm{p} 13$ & Ras association (RalGDS/AF-6) domain family member 2 & Negative & .039 \\
\hline
\end{tabular}

* A positive correlation reflects an association with an infiltrative phenotypic appearance, and a negative correlation reflects an association with an edematous appearance. 